\title{
Prevalência e severidade da cárie dentária em um município em condição de vulnerabilidade social no Estado do Paraná
}

\author{
Prevalence and severity of dental caries in a city in condition of social \\ vulnerability in the State of Paraná \\ Prevalencia y severidad de la caries dental en un municipio en condición de \\ vulnerabilidad social en el Estado de Paraná \\ Fernanda Midori TSUZUKI ${ }^{1}$ \\ Jéssica Catiste SILVA ${ }^{\mathbf{1}}$ \\ Larissa ISHIZU ${ }^{1}$ \\ Cristiane Muller CALAZANS ${ }^{2}$ \\ Mariliani Chicarelli da SILVA ${ }^{3}$ \\ Najara Barbosa da ROCHA \\ Departamento de Odontologia da Universidade Estadual de Maringá, UEM, 87020-900 Maringá - PR, Brasil \\ ${ }^{2}$ Departamento de Odontologia da Universidade Estadual de Maringá, UEM, 87020-900 Maringá - PR, Brasil \\ Mestre em Políticas Públicas pela Universidade Estadual de Maringá \\ Professora Associada, Departamento de Odontologia da Universidade Estadual de Maringá, UEM, 87020-900 Maringá - PR, Brasil \\ ${ }^{4}$ Professora Adjunto, Departamento de Odontologia da Universidade Estadual de Maringá, UEM, 87020-900 Maringá - PR, Brasil
}

\begin{abstract}
Resumo
Introdução: A doença cárie ainda é um grande problema de saúde pública que o Brasil enfrenta atualmente e crianças inseridas num contexto social economicamente desfavorecido podem ser grupos vulneráveis a esta doença. Objetivo: Identificar a prevalência, severidade da cárie dentária e necessidade de tratamento odontológico em crianças e jovens no município de Sarandi, PR. Métodos: A pesquisa é do tipo transversal, descritiva, retrospectiva, com dados quantitativos. A população do estudo compôs-se de crianças de 3 a 14 anos de idade, que frequentam um projeto voltado para o atendimento da população vulnerável, chamado Pescadores de Vida, no ano de 2016. Um treinamento com a equipe foi realizado, bem como, o estudo piloto, para ajuste do instrumento de coletas de dados e exame clínico. O termo de consentimento livre e esclarecido (TCLE) foi assinado pelas mães ou responsáveis. Os índices utilizados para coleta dos dados clínicos obedeceram aos critérios recomendados pela OMS, para levantamentos epidemiológicos. Resultados: Foi possível concluir que as crianças e adolescentes deste estudo têm alta prevalência de cárie dentária, bem como, necessidades de tratamentos complexos. Conclusão: Com isso, ressalta-se a necessidade de implantação de políticas sociais que contemplem as populações de menor nível socioeconômico.

Descritores: Cárie Dentária; Vulnerabilidade Social; Odontologia.
\end{abstract}

\begin{abstract}
Introduction: Caries disease is still a big public health problem facing Brazil and children in an economically disadvantaged social context may be vulnerable groups to this disease. Objective: Identify the prevalence, severity of dental caries and the need for dental treatment in children and young people in the city of Sarandi-PR. Methods: The research is of the transversal type, descriptive, retrospective, with quantitative data. The study population consisted of children from 3 to 14 years of age, who attend a project aimed at the care of the vulnerable population called Pescadores de vidas in the year 2016. A training with the researchers was carried out, as well as the pilot study to adjust the instrument of data collection and clinical examination. The written informed consent form (WICF) were signed by the mothers and guardians. The indices used to collect the clinical data obeyed the criteria recommended by World Health Organization for epidemiological surveys. Results: It was possible to conclude that the children and adolescents of this study have high experience and prevalence of dental caries as well as complex treatment needs. Conclusion: We conclude the need to implement social policies that contemplate the populations of lower socioeconomic level.

Descriptors: Dental Caries; Social Vulnerability; Dentstry.
\end{abstract}

\section{Resumen}

La enfermedad caries sigue siendo un gran problema de salud pública que Brasil enfrenta actualmente y los niños insertados en un contexto social económicamente desfavorecido pueden ser grupos vulnerables a esta enfermedad. Objetivo: Identificar la prevalencia, severidad de la caries dental y necesidad de tratamiento odontológico en niños y jóvenes en el municipio de Sarandi PR. Métodos: La investigación es del tipo transversal, descriptivo, retrospectivo, con datos cuantitativos. La población del estudio se compuso de niños de 3 a 14 años de edad, que frecuentan un proyecto orientado a la atención de la población vulnerable, llamado Pescadores de Vida, en el año 2016. Un entrenamiento con el equipo fue realizado, así como, el estudio piloto, para el ajuste del instrumento de recolección de datos y el examen clínico. El término de consentimiento libre y esclarecido (TCLE) fue firmado por las madres o responsables. Los índices utilizados para la recolección de datos clínicos se ajustaron a los criterios recomendados por la OMS para los estudios epidemiológicos. Resultados: Fue posible concluir que los niños y adolescentes de este estudio tienen alta experiencia y prevalencia de caries dentales, así como necesidades de tratamiento complejas. Conclusión: Con ello, se resalta la necesidad de implantación de políticas sociales que contemplen a las poblaciones de menor nivel socioeconómico.

Descriptores: Caries Dental; Vulnerabilidad Social; Odontología.

\section{INTRODUÇÃO}

Há indícios de que o arranjo da doença cárie nas populações é heterogêneo e fortemente associado à condição socioeconômica. Desse modo, persistentes desigualdades na condição bucal são encontradas entre os países e entre diferentes grupos sociais de um mesmo país ${ }^{1}$. Estudos sobre os determinantes sociais das doenças bucais têm ganhado importância e usualmente incluem fatores socioeconômicos, tais como o grau de escolaridade, a renda individual ou familiar e a ocupação dos indivíduos ${ }^{1-3}$.

A influência dos fatores contextuais na prevalência de cárie tem sido analisada nos últimos anos, incorporando-se indicadores socioeconômicos e ambientais, como renda per capita, Índice de Desenvolvimento Humano (IDH), Índice de
Gini, acesso aos serviços de saúde e disponibilidade de água

de abastecimento fluoretada. No Brasil, os inquéritos nacionais de saúde bucal mostram diferenças na distribuição da cárie, com carga de doença mais elevada nas regiões e nos grupos populacionais menos favorecidos economicamente ${ }^{3}$. A realidade social e econômica do município de Sarandi-PR é precária, na qual, muitas famílias se encontram em situação de vulnerabilidade social, o município possui uma incidência de pobreza de $48,7 \%$, sendo considerado o $18^{\circ}$ município mais pobre entre os municípios do estado do Paraná. Entre os municípios da região metropolitana de Maringá-PR, SarandiPR ocupa a primeira posição em incidência de pobreza ${ }^{4}$.

A atuação das universidades junto às comunidades revela uma oportunidade de auxiliar uma parte da população 
muitas vezes negligenciada. Por meio de orientações de higiene bucal, de dieta alimentar adequada e conscientização para redução de exposição a fatores de risco, visa-se estimular uma sensibilização geral da população para promoção da saúde e assim capacitar os indivíduos e a população a fim de obter controle sobre os fatores sociais e ambientais que afetam sua saúde bucal, para que, obtenham uma melhor qualidade de vida. Além disso, as atividades de extensão universitária possuem o objetivo de contribuir para formação de um profissional capaz de se sensibilizar as necessidades de saúde da população e realizar a integração multidisciplinar, como por exemplo, ações de natureza preventiva e curativa, teoria e prática, e entre ensino e serviço ${ }^{5}$.

No entanto é necessária a realização de um levantamento epidemiológico para avaliar qual a real situação da saúde bucal dessa população, a fim de possibilitar o melhor enfoque para sua atenção odontológica e promoção de saúde bucal. Portanto, o objetivo deste estudo foi identificar a prevalência, severidade da cárie dental e necessidade de tratamento odontológico na população de 3 a 14 anos de idade no município de Sarandi-PR, em condição de vulnerabilidade social no Estado do Paraná, de um projeto voltado para a população carente.

\section{MATERIAL E MÉTODO}

A pesquisa é do tipo transversal, descritiva, retrospectiva, com dados quantitativos, realizada no município de Sarandi, localizada no noroeste do Paraná, com uma população estimada de 91.344 habitantes no ano de $2016^{4}$. O município apresenta indicadores socioeconômicos de saúde abaixo de todo o Estado do Paraná. Esta cidade não possui fluoretação nas águas de abastecimento público e há escasso atendimento odontológico no serviço público de saúde.

A população do estudo compôs-se de crianças e jovens que frequientaram um projeto voltado para o atendimento da população vulnerável chamado Pescadores de Vida, durante o ano de 2016. Como critérios de inclusão foram considerados: crianças e adolescentes que estavam entre a idade de 3 a 14 anos de idade, que permitiram realizar o exame clínico; mães ou responsáveis que após explicação e elucidação da pesquisa assinaram o termo de consentimento livre e esclarecido (TCLE). Foram excluídas as crianças que se recusaram realizar o exame clínico ou os responsáveis não quiseram participar da pesquisa.

Um treinamento com a equipe foi realizado, bem como o estudo piloto, para ajuste do instrumento de coletas de dados e exame clínico da crianças e adolescentes.

Os índices utilizados para coleta dos dados clínicos obedeceram aos critérios recomendados pela Organização Mundial de Saúde (OMS), utilizando-se espelho bucal plano e a sonda CPI para levantamentos epidemiológicos, sob luz natural, com o examinador e paciente sentados ${ }^{6}$. Para a dentição decídua utilizou-se o índice ceo-d, representativo do número de dentes cariados (c), com extração indicada (e) e obturados (o), segundo a unidade dente. Para a dentição permanente utilizou-se o índice CPO-D, representativo do número de dentes cariados $(\mathrm{C})$, com extração indicada ou extraídos $(\mathrm{P})$ e obturados $(\mathrm{O})$, segundo a unidade dente ${ }^{6}$.

Os dados foram digitados em um banco de dados e analisados pelo software EpiInfo. A análise estatística incluiu a análise descritiva e inferencial, com nível de significância de $p \leq 0,05$ e intervalo de confiança (IC) de $95 \%$.

Foram respeitados os aspectos éticos para a realização de pesquisas com seres humanos e a declaração de Helsinki, bem como a aprovação no Comitê de Ética e Pesquisa em Seres Humanos da Universidade Estadual de Maringá (CAAE 66295817.8.0000.0104).

\section{RESULTADOS}

O tamanho da população analisada foi de 53 jovens e crianças $(n=53)$, com idades entre 3 e 14 anos, sendo que $35,8 \%$ são do sexo masculino e $64,2 \%$ sexo feminino (Tabela 1).

Os resultados mostraram que a população estudada teve alta prevalência da doença cárie, ou seja, 88,7\% dos examinados apresentavam a doença. Apenas 6 crianças $(11,3 \%)$ estavam livres da doença cárie atualmente.

Tabela 1. Distribuição numérica e percentual com relação à faixa etária e gênero analisados

\begin{tabular}{|l|l|l|}
\hline Faixa etária & $\mathbf{n}$ & \% \\
\hline 3 a 4 anos & 2 & 3,7 \\
\hline 5 anos & 4 & 7,5 \\
\hline 6 aos 11 anos & 35 & 66 \\
\hline 12 anos & 5 & 9,4 \\
\hline 13 a 14 anos & 7 & 13,2 \\
\hline Total & 53 & 100 \\
\hline Sexo & $\mathbf{n}$ & $\%$ \\
\hline Masculino & 19 & 35,8 \\
\hline Feminino & 34 & 64,2 \\
\hline Total & 53 & 100 \\
\hline
\end{tabular}

O índice de cárie média (CPO-D ou ceo-d) da população estudada foi 5 e apenas 2 crianças $(3,8 \%)$ não tiveram nenhuma experiência de cárie. O índice aos 3 e 4 anos de idade foi de 8 . Aos 5 anos foi de 6,75. Dos 6 até os 11 anos de idade o índice decresceu para 4,85. Já aos 12 anos o índice cpo-d foi de 6 e a partir dos 13 anos, o valor foi de 5,25 (Gráfico 1).

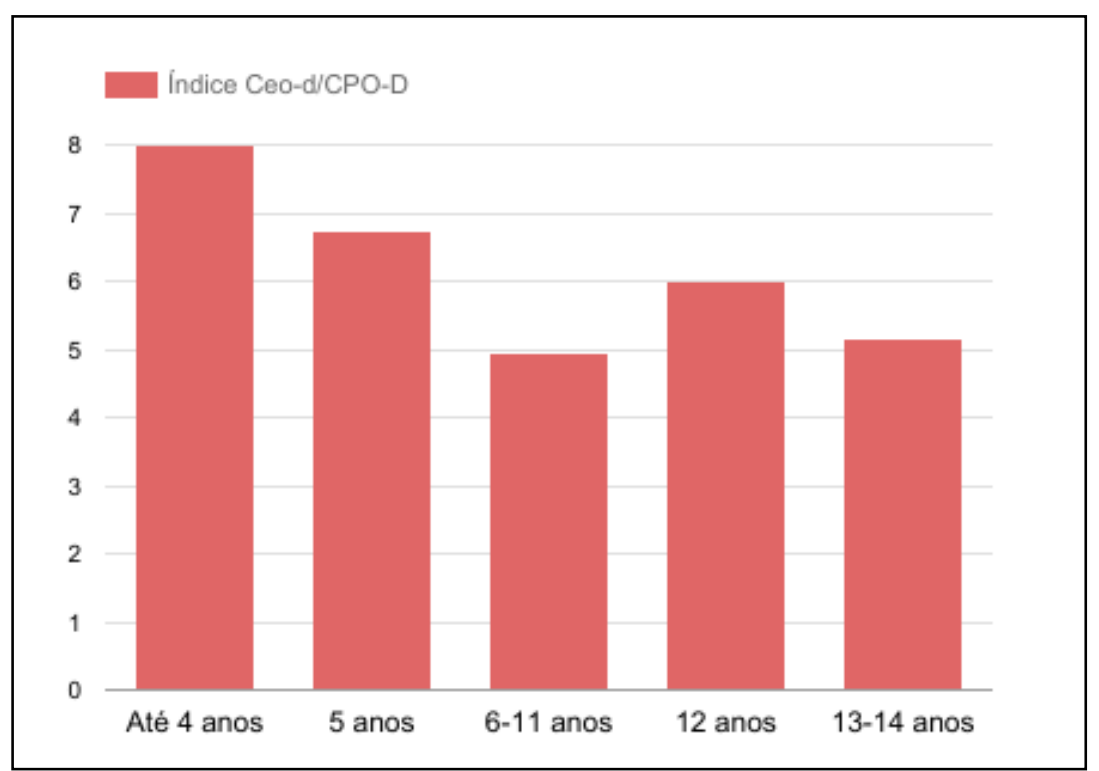

Gráfico 1: Distribuição numérica dos índices CEO-D e CPO-D de acordo com idade.

Dentre os dentes avaliados, 108 apresentam-se cariados com 32 dentes que apresentam situação crítica, com necessidade de tratamento de endodontia ou exodontia. A prevalência de cárie na dentição decídua aos 5 anos de idade foi de $100 \%$. A severidade da doença, representada pelo índice Ceo-d, foi de 6,75. A prevalência de cárie na dentição permanente aos 12 anos de idade foi de $100 \%$, e a severidade da doença, representada pelo índice CPO-D, foi de 6 .

\section{DISCUSSÃO}

Os dados mostram que a prevalência e a severidade da cárie, bem como as necessidades de tratamento da população estudada foram muito elevadas. O índice CPO-D e ceo-d das crianças e jovens apresentou-se acima da média brasileira. A 
situação socioeconômica dessas famílias pode ser considerada como um dos fatores, agravado pela ausência de fluoretação nas águas de abastecimento público da cidade de Sarandi-PR e que existem poucos profissionais dentistas disponíveis na atenção básica para a população.

$\mathrm{O}$ índice CPO-D verifica o ataque de cárie por dente e foi originalmente formulado em $1937^{7}$. Ele continua sendo o índice mais utilizado em todo o mundo e serve de referência para o diagnóstico das condições dentais e para formulação e avaliação de programas de saúde bucal. Na região Sul do país, os dados do levantamento epidemiológico em saúde bucal realizado em 2010, indicaram aos cinco anos de idade, um índice CPO-D de 2,56 e aos doze anos de idade, 2,17 nas cidades do interior. Em todo o Brasil, a média aos cinco anos de idade é de 2,43 e aos doze anos $2,07^{8}$. Este estudo sugere que a situação epidemiológica de cárie dentária nas crianças e adolescentes está muito elevada. Os dados encontrados colocam o município como um dos mais altos índices registrados no Brasil.

A cárie dentária é uma doença crônica e constitui um dos grandes problemas de saúde pública no Brasil atingindo indivíduos de todas as faixas etárias e níveis socioeconômicos. É uma patologia multifatorial, infecciosa, que ocasiona a desmineralização das estruturas dentárias produzida pela ação de ácidos provenientes do metabolismo de carboidratos na placa bacteriana dentária. Um estudo realizado em todo o Brasil, buscando correlacionar os determinantes individuais e contextuais da cárie em crianças brasileiras de 12 anos em 2010, apontam que os piores indicadores dessa doença foram observados em crianças de famílias com renda mais baixa e que viviam em municípios com piores indicadores econômicos e sem acesso à água fluoretada ${ }^{10}$, como verificado no município estudado.

A doença cárie vem diminuindo mundialmente, porém em algumas localidades existem altos níveis de cárie devido aos determinantes sociais. Esse fenômeno é conhecido como "polarização", quando num polo há ausência de doença e, no outro, um grande número de casos concentrados num pequeno grupo de indivíduos. Ao analisar indicadores de saúde no Brasil, foi percebido que embora a expectativa de vida tenha aumentado e os valores dos coeficientes de mortalidade geral, e por causas específicas, tenham diminuído nas últimas décadas, persistem importantes diferenças entre as regiões e variações segundo o nível de renda ${ }^{11}$. Esse aspecto sugere um outro fenômeno, o da iniquidade, em que, no caso da cárie, o ataque desigual da doença entre os indivíduos decorre não apenas de variações biológicas inevitáveis, mas também das diferenças que têm origem na ordem social onde estão inseridos, e que se expressam por meio do processo saúde-doença ${ }^{3,12}$.

O IDH é considerado um potente avaliador das condições sociais e ambientais, refletindo a renda e a educação, além de indicadores extremamente sensíveis como a mortalidade infantil, que foi demonstrado uma correlação entre os altos valores do IDH e baixos valores de experiência de cárie $^{13}$. O município de Sarandi apresenta um IDH de $0,695^{14}$. Esse índice, considerado baixo demonstra uma menor qualidade de vida que pode estar interferindo com os fatores determinantes da doença cárie.

O acesso à água fluoretada é claramente influenciado por condições socioeconômicas, pois são os municípios mais pobres que têm mais dificuldade em implantar a medida. $\mathrm{Na}$ região Sul, 4,9\% dos municípios não tem fluoretação nas águas. No Paraná, a fluoretação nas águas começou quando o abastecimento público era atendido pelo Departamento de Água e Esgoto (DAE), vinculado à Secretaria de Viação e Obras do Estado. A partir de 1972 os sistemas estaduais foram assumidos pela Sanepar e somente em 24 de maio de 1974, pela Lei Federal n. ${ }^{\circ} 6050^{15}$, tornou-se obrigatória a fluoretação das águas de abastecimento em todo o território brasileiro. Esta lei foi regulamentada pelo Decreto Federal de n. ${ }^{\text {o }} 76.872^{16}$, de 22 de dezembro de 1975 . No entanto, até os dias atuais, o município de Sarandi-PR não conta com água fluoretada ${ }^{17}$.

A possibilidade de intervir na dissolução do esmalte dentário, diminuindo significativamente a perda de minerais e de reverter o progresso de lesões cariosas iniciais torna $o$ fluoreto um elemento indispensável à saúde dental. Enquanto recurso preventivo, o fluoreto é uma alternativa de saúde pública bucal extremamente eficaz e de baixo custo ${ }^{18}$. O flúor é altamente eficaz no combate à cárie dentária e também estudos realizados pela OMS revelaram que a cada 1 dólar investido em fluoretação são economizados 50 dólares, que seriam necessários aos processos de tratamento dentário e às despesas indiretas ${ }^{17}$. Segundo o Ministério da Saúde, a aplicação do produto possibilita, no mínimo, uma redução de $65 \%$ das cáries, desde que mantida a continuidade e a regularidade dos teores de flúor na água ${ }^{19}$. Esta seria uma alternativa viável de promoção de saúde bucal nesta população vulnerável na ausência de fluoretação das águas.

O desafio de promover a saúde bucal é particularmente grande em grupos populacionais socioeconômicos desfavorecidos, pois as condições precárias de vida estão associadas à disponibilidade e acessibilidade limitada a serviços de saúde bucal, e a outros fatores de risco ${ }^{20}$. Segundo a Lei Orgânica da Saúde ${ }^{21}$, o acesso universal e gratuito aos serviços e ações de saúde devem ser preconizados, como por exemplo, a utilização dos serviços odontológicos. No entanto, apesar da importância da saúde bucal ser reconhecida, parte da população brasileira não tem acesso aos serviços de saúde bucal $^{22}$, consequentemente a atenção bucal está longe da universalização e equidade, considerados princípios do Sistema Único de Saúde (SUS) ${ }^{23}$. Na literatura, a percepção da população sobre a importância da saúde bucal pode estar associado ao acesso ao serviço odontológico ${ }^{24}$.

Em 2014, foi publicada a linha guia no Paraná a fim de permitir uma homogeneização de conceitos e de processos na busca de um trabalho mais efetivo e eficiente, com foco na qualidade da atenção e na humanização ${ }^{25}$. Foi estabelecida uma meta de $100 \%$ das crianças nascidas em 2015 chegar aos 5 anos de idade, em 2020, com zero cárie. Para que essa meta seja atingida, a consciência com relação à alimentação, amamentação, limpeza e escovação, e hábitos devem ser bem estabelecidas em todo o Estado. No entanto, os índices apresentados neste estudo mostram que será difícil esta meta ser alcançada, tendo em vista os elevados índices de cárie apresentados.

A importância dos fatores sociocomportamentais e ambientais na saúde bucal tem sido relatada em trabalhos socioepidemiológicos ${ }^{26}$. Os fatores determinantes de doenças bucais são bem conhecidos, são fatores de risco comuns a um grupo de doenças crônicas: dieta, má higiene, tabagismo, alcoolismo e condutas de risco que podem gerar lesões. Estudos mostram que criar ações e investimentos na promoção de saúde bucal e em cuidados preventivos, pode reduzir a prevalência de doenças bucais ${ }^{20,26}$, e a criação de condições que possibilitem aos indivíduos e a comunidade desfrutarem de uma boa saúde bucal é um desafio.

A melhoria da situação de saúde bucal implica em transformações sociais que promovam impacto nas condições de vida existentes, repercutindo, de forma positiva, em várias vertentes: trabalho, moradia, educação, renda, acesso a serviços de saúde. Por outro lado, ações específicas de promoção de saúde bucal podem e devem ser implantadas elou implementadas, no plano microestrutural, seja no âmbito dos serviços de saúde, ou no ambiente de aprendizado. Ações de educação em saúde aos pais e responsáveis, ampliar o acesso aos serviços de saúde promovendo ações de diagnóstico e tratamento segundo risco de cárie e ações de 
prevenção com visitas periódicas na Atenção Básica devem ser reforçadas ${ }^{27,28}$.

Intervenções educativas e preventivas focalizadas em grupos mais acometidos podem ser eventualmente necessárias para o enfrentamento dos problemas bucais ${ }^{29}$. Perante essa situação, o projeto de extensão da Universidade Estadual de Maringá Pequenos gestos, grandes sorrisos (Proc $\mathrm{n}^{\circ}$ 4330/2008), se preocupa em levar todas as informações necessárias para esta parcela populacional de Sarandi-PR sobre os assuntos que envolvam a manutenção da saúde bucal, utilizando como meio de comunicação, palestras e mídias sobre temas que geram problemas freqüentes. Algumas ações coletivas desse projeto são realizadas em conjunto com os voluntários do Projeto Pescadores de Vida na cidade de Sarandi- PR, que realizam trabalhos população deste estudo, considerada socialmente vulnerável, com o objetivo resgatar as crianças para se tornarem meios de transformação da realidade social na qual vivem. Com as crianças são desenvolvidas atividades voltadas para a área lúdica, porém com a mesma finalidade: instruir sobre os cuidados que devem ser tomados para se obter uma boa saúde bucal. Além do atendimento curativo em ambiente domiciliar são realizadas aplicações tópicas de flúor, escovação supervisionada e educação em saúde.

Para promover saúde não é suficiente informar, é necessária uma relação dialogal, em que os sujeitos sejam envolvidos em todos os momentos da ação educativa, levando em conta a reconstrução do saber da escola e a formação continuada dos docentes ${ }^{30}$. Deve-se aplicar os conhecimentos científicos disponíveis há tantas décadas, destacando-se a importância do trabalho da Universidade juntamente com a comunidade, na área de promoção da saúde bucal, a fim de garantir qualidade de vida para os brasileiros. Os resultados deste estudo foram significativos, porém devem-se ressaltar algumas limitações deste estudo. A primeira limitação foi sobre o desenho do estudo ser do tipo transversal e por isso ter alguns vieses, havendo a necessidade de realização de estudos longitudinais a partir destes dados obtidos. A outra seria sobre a metodologia utilizada que foi na forma de entrevista. Contudo considera-se que os resultados deste estudo possam contribuir para o planejamento de ações de promoção de saúde bucal em populações vulneráveis.

\section{CONCLUSÃO}

Com os resultados sugerem que as crianças e adolescentes deste estudo tem alta experiência e prevalência de cárie dentária, bem como a urgência de tratamentos complexos. Com isso, ressalta a necessidade de implantação de políticas sociais que contemplem as populações de menor nível socioeconômico e em regiões vulneráveis de forma direcionada, com o intuito de diminuir o diferencial nos indicadores do processo saúde-doença. Para isso, as políticas de saúde pública devem ter como prioridade a incorporação de medidas que incentivem os bons hábitos de saúde e a criação de ambientes saudáveis, com vistas a promover a saúde desta população e qualidade de vida. A relação universidade-comunidade deve ser explorada e incentivada para diminuir os índices da doença cárie em comunidades de vulnerabilidade social.

\section{REFERÊNCIAS}

1. Petersen PE, Kwan S. Social determinants and public health programmes--the case of oral health. Community Dent Oral Epidemiol. 2011; 39(6):481-7.

2. Boing AF, Peres MA, Kovaleski DF, Zange SE, Antunes JLF. Sócio-econômica em estudos epidemiológicos de cárie dentária e doenças periodontais: características da produção na década. Rev Saúde Pública 2005; 21(3):673-8.
3. Narvai PC, Frazão P, Roncalli AG, Antunes JLF. Cárie dentária no Brasil: declínio, polarização, iniqüidade e exclusão social. Rev Panam Salud Publica 2006; 19(6):385-93.

4. IBGE: Censo Demográfico. Brasil. [Internet]. [Acesso em: 30 de agosto de 2016]. Disponível em: http://www.ibge.gov.br/home/

5. Costa ICC, Unfer B, Oliveira AGRC, Arcieri RM, Saliba NA. Integração universidade-comunidade: análise das atividades extra-murais em odontologia nas universidades brasileiras. Rev Cons Reg Odontol Minas Gerais 2000; 3(6):146-53.

6. World Health Organization. [Internet] 1997. Oral health survey: basic methods. 4th ed. Geneva. [Acesso em 18 de agosto de 2017] Disponível em: http://www2.paho.org/hq/dmdocuments/2009/OH_st_Esu rv.pdf.

7. Klein H, Palmer CE. Dental caries in American indian children. Publ. Hlth Bull. 1937 dec; 239:1-54.

8. Brasil. Ministério da Saúde. Projeto SB Brasil 2010 [Internet]. Brasília: Pesquisa Nacional de Saúde Bucal. Resultados Principais. [Acesso em: 23 de agosto de 2017]. Disponível http://dab.saude.gov.br/CNSB/sbbrasil/.

9. Lima JE. Cárie dentária: um novo conceito. R Dental Press Ortodon Ortop Facial. Maringá 2007; 12 ( 6):119130.

10. Freire MCM, Reis SCGB, Figueiredo N, Peres KG, Moreira RS, Antunes JLF. Determinantes individuais e contextuais da cárie em crianças brasileiras de 12 anos em 2010. Rev Saúde Pública 2013; 47(Supl 3):40-9.

11. Almeida C, Travassos C, Porto S, Labra ME. Health sector reform in Brazil: a case study of inequity. Int $\mathbf{J}$ Health Serv. 2000; 30(1):129-62.

12. Silva MAM, Souza MCA, Rodrigues CRT, Bello RF. Condições de saúde bucal em escolares de Vassouras/RJ: uma pesquisa epidemiológica. Rev Brasil Pesq Saúde 2010; v. 12, n.1, p.52-56.

13. Frias AC, Antunes JLF, Junqueira SR, Narvai PC. Determinantes individuais e contextuais da prevalência de cárie dentária não tratada no Brasil. Rev Panam Sauld Publica 2007; 22(4):279-285.

14. Atlas Brasil 2013 [Internet] Brasil: Programa das Nações Unidas para o Desenvolvimento Humano - PNUD. [Acesso em: 21 de agosto de 2017]. Disponível em: http://atlasbrasil.org.br/2013/.

15. Brasil. Lei Federal n. ${ }^{\circ}$ 6050. Dispõe sobre a fluoretação da água em sistemas de abastecimento quando existir estação de tratamento. Diário Oficial da República Federativa do Brasil, 1974.

16. Brasil. Decreto Federal n. ${ }^{\circ} 76.872$ - Regulamenta a Lei ${ }^{\circ}$ 6.050 , de 24 de maio de 1974, que dispõe sobre a fluoretação da água em sistemas públicos e abastecimento. Diário Oficial da República Federativa do Brasil, 1975.

17. Araújo IC, Aragão MV, Melo CB, Araújo JL, Barroso RF. Fluoretação das águas dos sistemas de abastecimentos públicos. J Asses Odontol 2002; 5 (32):24-25.

18. Araújo MT, Campos EJ, Rodrigues CS, Serravalle LS, Lima MJ, Araújo, DB. Ação do fluoreto de dentifrícios sobre o esmalte dentário. Rev Ci Méd Biol. 2002; 1(1):16-32.

19. Narvai PC. Cárie dentária e flúor: uma relação do século XX. Cienc Saude Coletiva 2000; 5(2):381-92.

20. Petersen PE. The World Oral Health Report 2003: continuous improvement of oral health in the $21 \mathrm{st}$ century--the approach of the WHO Global Oral Health Programme. Community Dent Oral Epidemiol. 2003; 31(Suppl 1): 3-23 
21. Brasil. Lei orgânica de saúde $n^{\circ}$. 8.080. Diário Oficial da República Federativa do Brasil, 1990.

22. Ferreira AAA, Piuvezam G, Werner CWA, Alves MSCF. A dor e a perda dentária: representações sociais do cuidado à saúde bucal. Ciênc Saúde Coletiva 2006; 11(1):211-8

23. Gomes PR, Costa SC, CyprianoS, Sousa MLR. Paulínia, São Paulo, Brasil: situação da cárie dentária em relação às metas OMS 2000 e 2010. Cad Saúde Pública 2004; 20(3):866-70.

24. Sanders AE, Slade GD. Deficits in perceptions of oral health to general health in populations. J Public Health Dent. 2006; 66(4):255-62.

25. Brasil. Secretaria de Estado da Saúde do Paraná. Curitiba: Superintendência de Assistência à Saúde. Linha guia de saúde bucal - SESA, 2014.

26. Sheiham AA. Determinação de necessidades de tratamento odontológico: uma abordagem social. In: Pinto VG. Saúde bucal coletiva. p.222-50. 4th ed. São Paulo: Ed. Santos, 2000

27. Olatosi OO, Inem V, Sofola OO, Prakash P, Sote EO. The prevalence of early childhood caries and its associated risk factors among preschool children referred to a tertiary care institution. Niger J Clin Pract. 2015; 18(4): 493-501.

28. Stephen A, Krishnan R, Ramesh M, Kumar VS Prevalence of early childhood caries and its risk factors in 18-72 month old children in Salem, Tamil Nadu. J Int Soc Prev Community Dent. 2015; 5(2):95-102.

29. Lemkuh I, Souza MVC, Cascaes AM, Bastos JL. The effectiveness of educational interventions in oral health: a literature review. Cad. Saúde Colet. 2015; 23(3):336-46 345.

30. Vasel J, Bottan ER, CAMPOS L. Educação em saúde bucal: análise do conhecimento dos professores do ensino fundamental de um município da região do Vale do Itapocu (SC). Rev Sul Brasil. 2005; 5(2):12-18.

\section{CONFLITO DE INTERESSES}

Os autores declaram não haver conflitos de interesse.

\section{AUTOR PARA CORRESPONDÊNCIA}

\section{Fernanda Midori Tsuzuki}

fertsuzuki@gmail.com

Submetido em 08/02/2018

Aceito em 20/02/2018 